\title{
Optimization Models for Fixed Channel Assignment in Wireless Mesh Networks with Multiple Radios
}

\author{
Arindam K. Das, Hamed M. K. Alazemi, Rajiv Vijayakumar, Sumit Roy
}

\begin{abstract}
The combination of multiple radio nodes in conjunction with a suitably structured multi-hop or mesh architecture has the potential to solve some of the key limitations of present day wireless access networks that are based on single-radio nodes. This paper addresses the channel assignment problem for multi-channel multi-interface (radio) wireless mesh networks. We focus on static wireless mesh networks where multiple nonoverlapping channels are available for each wireless interface. In this network environment, our objective is to find a fixed channel assignment which maximizes the number of bidirectional links that can be activated simultaneously, subject to interference constraints. We present two mixed integer linear programming models for solving the fixed channel assignment problem with multiple radios. Detailed computational results on various grid topologies are also presented and discussed.
\end{abstract}

\section{INTRODUCTION}

The emergence of cost-effective wireless access networking technology such as 802.11 has changed mobile communications and computing in significant ways. Its success to date has been largely due to its deployment in the home and small enterprise segments where it has limited coverage and serves only a few users simultaneously. However there is already good evidence that the current dominant model of user access based on the infrastructure mode of 802.11 is poorly suited for the dense AP networks which will be needed in order to allow the number of users to scale. This is, in part, due

A.K. Das is a joint Post Doctoral Research Associate at the Department of Electrical Engineering and the Department of Aeronautics and Astronautics, University of Washington, Box 352500, Seattle, WA 98195. e-mail: arindam@ee.washington.edu.

H. Alazemi is an Assistant Professor at the Department of Computer Engineering at Kuwait University. $\mathrm{He}$ is currently a visiting faculty at the Department of Electrical Engineering, University of Washington, Box 352500, Seattle, WA 98195. e-mail: hamed@ee.washington.edu

R. Vijayakumar is a Post Doctoral Research Associate at the Department of Electrical Engineering, University of Washington, Box 352500, Seattle, WA 98195.e-mail: rajiv@ee.washington.edu.

S. Roy is with the Department of Electrical Engineering, University of Washington, Box 352500, Seattle, WA 98195. e-mail: roy@ee.washington.edu. to architectural reasons as well as inherent protocol stack design limitations.

It is well-known from cellular systems engineering that the key to one-hop capacity scaling (such as between a client and AP) is enhancing spatial reuse, i.e., reducing the re-use distance between co-channel users on different AP's. In narrow-band systems, the extent of spatial reuse is directly proportional to the number of orthogonal channels (or system bandwidth) available. Currently, only a very limited number of such orthogonal channels are available: 3 in $802.11 \mathrm{~b}(2.4 \mathrm{GHz})$ and 12 in $802.11 \mathrm{a}(5 \mathrm{GHz})$. Although greater worldwide allocation is anticipated for unlicensed use in the future, it is clear that relying primarily on increased available bandwidth for scaling is not a feasible option. Accordingly, for any given system bandwidth, optimizing network performance necessarily requires improving the entire protocol stack.

A promising option for scaling the capacity of a wireless access network is to configure a Layer- 2 mesh as is being currently planned within the IEEE 802.11s Task Group. This implies a direct wirelessly inter-connected set of mesh nodes (which comprise Access Points that allow direct client access and future 'routers' that only relay packets between other mesh elements) to form a multi-hop network. The ad-hoc (but static) nature of mesh node deployments results in a significant spatial variability of the multi-access interference (MAI) seen at any node location, implying variable location-dependent node throughput. Hence, effective topology modification mechanisms including power control, node clustering and channel assignments are anticipated to be important design degrees of freedom.

Traditional multi-hop wireless networks (also called packet radio networks) have almost exclusively comprised of single radio elements. For various reasons, including notably the following discussion on channel assignment for a single radio mesh, such networks are unable to effectively scale to exploit the increasing system bandwidth available. Consequently, the use of multiple radio nodes in a mesh network appears to provide the most promising approach to overcoming the above problems. Multiple radios greatly increase 
the potential for enhanced channel selection and route formation while the mesh allows more fine-grained interference management and topology (power) control. This paper addresses the channel assignment problem for multi-channel multi-radio wireless mesh networks. We focus on static wireless mesh networks where multiple non-overlapping channels are available for each wireless interface.

There are a number of common issues involved in traditional multi-hop wireless networks. These, as was noted in [1] and [2], include efficient methods for sharing the common radio channel, network connectivity, network capacity, and methods for managing and controlling the distributed network. A particular issue that is of interest to us is the channel assignment problem in multi-hop wireless networks with a single radio. This issue has been subject to several studies in the literature. Early work by Cidon et al. [3] presented a distributed dynamic channel assignment algorithm that is suitable for shared channel multi-hop networks. Hajek and Sasaki [4] studied the network as an arbitrary undirected graph and presented two polynomial time algorithms for link scheduling. They also addressed the problem of joint routing and scheduling to satisfy end-toend demand. They showed that, under a certain simplifying assumption, the routing and scheduling problems can be decoupled to a large extent, without increasing the schedule length. Their algorithm complexity was later improved by Ogier in [5] with a more efficient approach. When using an arbitrary graph modeling approach, many scheduling problems were shown by researchers to be NP-hard [6], [7], [8].

A natural way to increase network capacity and utilization is by exploiting the use of multiple channel and channel reuse opportunities. Several studies on the subject of multi channel multi-hop wireless networks have been the main subject of research in recent years. In [9], [10], [11], [12], for example, MAC protocols based on modification of IEEE 802.11 were proposed for utilizing multiple channels. In particular, Jain et al. [9] propose a protocol that selects channels dynamically and employs the notion of "soft" channel reservation. This reservation based scheme, which was later extended in [10], gives preference to the channel that was used for the last successful transmission. So et al. [11] propose a MAC protocol which enables hosts to dynamically negotiate channels such that multiple communication can take place in the same region simultaneously, each in different channel. The proposed scheme requires only a single transceiver for each host. They later extend their study in [12] to propose a routing protocol for multi-channel multi-hop wireless networks with a single interface that finds routes and assigns channels to balance load among channels while maintaining connectivity.

While the above studies requires modification to the IEEE 802.11, Bahl et al. [13] propose a new protocol, Slotted Seeded Channel Hopping ( $\mathrm{SSCH}$ ), that extends the benefits of channelization to ad-hoc networks. Their protocol runs over unmodified IEEE 802.11 with a single interface. The $\mathrm{SSCH}$ protocol operates at the link layer, but it can be implemented in software over an IEEE 802.11-compliant wireless network interface card. They show through extensive simulations that their proposed scheme yields significant capacity improvement in a variety of single hop and multi-hop wireless scenarios.

A few approaches to the routing and channel assignment problems in multi-hop multi-radio mesh networks have been proposed [14], [15], [16], [17], [18]. In particular, Raniwala et al. [17], [18] propose a centralized load-aware joint channel assignment and routing algorithm, which is constructed with a multiple spanning tree-based load balancing routing algorithm that can adapted to traffic load dynamically. They demonstrate the dependency of the channel assignment on the load of each virtual link, which in turns depends on routing. They also show that the problem of channel assignment is NP-hard.

Kyasanur et al. [16] studied the multi-radio mesh network under the assumption that the network has the ability to switch an interface from one channel to another dynamically. They present a distributed interface assignment strategy that accounts for the cost of interface switching and does not make any assumptions on the traffic characteristics. Their routing strategy selects routes which have low switching and diversity cost taking into account the global resource usage to maximize the network utilization and allows the nodes to communicate without any specialized coordination algorithm.

A different aspect of the multi-hop multi-radio mesh networks was investigated by Draves et al. [15]. They propose a new routing metric (WCETT) which is implemented in a routing protocol called Multi-Radio LinkQuality Source Routing (MR-LQSR), in which all nodes are assumed to be stationary and the channel assignment is predetermined. The WCETT metric is aimed at achieving a tradeoff between delay and throughput by balancing the usual criterion (minimum number of hops) with the notion of channel diversity. Their results show that classical shortest path routing is not suitable when multiple radios are deployed; exploiting channel diversity in multi-radio mesh networks significantly improves the network capacity and makes better utilization of the channel resources. 
In this paper, we consider the problem of static channel assignment in multi-hop multi-radio mesh networks and discuss two integer linear programming (ILP) models which can be used for solving the problem optimally. Our objective function is maximization of the number of links that can be active simultaneously.

The remainder of the paper is organized as follows. We discuss our network model and assumptions in Section II and formally define the problem in Section III. In Sections IV and V, we discuss two different ILP models for solving the channel assignment problem with multiple radios. Section VI provides numerical simulation results for various sizes of grid topologies. Section VII concludes the paper.

\section{Network Model And Assumptions}

In this section, we outline our network model and assumptions.

1) We consider a fixed $N$-node wireless network.

2) Each node has $K$ radios, $K \geq 2$.

3) Given a target probability of bit error rate from node $i$ to node $j$, the transmitter power at node $i$ necessary to support the link $i \rightarrow j,{ }^{1} \mathbf{P}_{i j}$, is proportional to $P_{0} d_{i j}^{\alpha}$, where $d_{i j}$ is the Euclidean distance between nodes $i$ and $j, \alpha$ is the channel loss exponent, typically between 2 and 4 , and $P_{0}$ is the reference power when $d_{i j}=1 \mathrm{~m}$. Without any loss of generality, we set the proportionality constant equal to 1 and therefore:

$$
\mathbf{P}_{i j}=P_{0} d_{i j}^{\alpha}
$$

4) We assume that $\mathbf{P}_{i j}=\mathbf{P}_{j i}, \forall(i, j) \in \mathcal{N}$ where $\mathcal{N}$ is the set of all nodes in the network and $N=|\mathcal{N}|$.

5) There is a constraint on the maximum power level per sector which a node can use for transmission and that this parameter is identical for all nodes. We denote this maximum power level by $P^{\max }$.

6) Let $\mathcal{E}$ be the set of all bidirected edges in the network and $E=|\mathcal{E}|$. Using the transmitter power constraint, the set $\mathcal{E}$ is given by:

$$
\mathcal{E}=\left\{(i \leftrightarrow j):(i \neq j) \in \mathcal{N}, P^{\max } \geq \mathbf{P}_{i j}\right\}
$$

The third condition on the right hand side of (2) enforces bidirectionality of edges based on the maximum power constraint. Note that because of our assumption that the matrix $\mathbf{P}$ is symmetric, $P^{\max } \geq \mathbf{P}_{i j} \Rightarrow P^{\max } \geq \mathbf{P}_{j i}$. For the sake of notational simplicity, we will also use the set $\mathcal{E}$ to

\footnotetext{
${ }^{1}$ We use the notation $\{i, j\}$ to represent the node pair. A directed link from $i$ to $j$ will be represented by $i \rightarrow j$ and an undirected (or, bidirected) link between $i$ and $j$ will be represented by $i \leftrightarrow j$.
}

refer to all directed edges, $\{i \rightarrow j\}$, in the graph, since:

$$
(i \leftrightarrow j) \in \mathcal{E} \Leftrightarrow(i \rightarrow j) \in \mathcal{E} \&(j \rightarrow i) \in \mathcal{E}
$$

We will refer to the graph $G=(\mathcal{N}, \mathcal{E})$ as the reachability graph of the network.

\section{PROBLEM Formulation}

We consider the static channel assignment problem on a network of $N$ nodes, each of which has $K$ radios. Let $F$ denote the number of available orthogonal channels, $F>1$. Given an assignment of channels to radios, nodes $i$ and $j$ can communicate if and only if they share a common channel and are within communication range of each other. If these conditions are satisfied, we say that a link exists between $i$ and $j$. One of the goals of the channel assignment problem is to ensure network connectivity. The approach we will adopt in this paper is to require that if two nodes are within communication range of each other, they must share a common channel for data transmission and reception. An assignment which satisfies this property ensures network connectivity and is said to be feasible.

Typically there will be many feasible channel assignments and we would therefore like an optimality criterion that allows us to pick one of these channel assignments. The optimality criterion we use is maximization of the number of possible simultaneous transmissions in the network. The rationale behind this choice is explained below.

We assume that the nodes in the mesh employ the RTS/CTS mechanism to combat the hidden terminal problem that arises naturally in the context of the relatively large distances involved in the mesh architecture. If a single channel is available, after a successful RTS/CTS exchange between a pair of nodes, no node within communication range of either the sender or the receiver can communicate for the duration of the subsequent data packet. Figure 1 shows the set of links that must remain silent (if they are on the same channel) when the edge $(a \leftrightarrow b)$ is active, given that the set of nodes forms a grid topology. For this topology, the set contains 22 edges. This illustrates that an ongoing transmission can potentially silence a large number of other links if they share the same channel, which motivates the choice of a channel assignment which maximizes the number of simultaneous transmissions as our optimality criterion. Intuitively, such an assignment should maximize the 1hop (link layer) throughput (as opposed to multihop end-to-end throughput) in the network in worst case traffic; i.e., when the traffic profile is such that there 


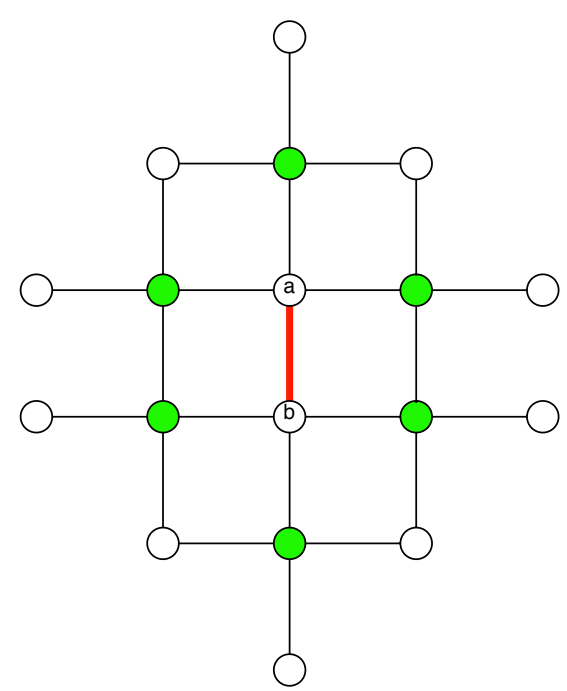

Fig. 1. Set of 22 interfering edges for the edge $a \leftrightarrow b$ (in bold), if all are on the same channel. The shaded nodes are neighbors of either node $a$ or node $b$ and must remain silent when the link $a \leftrightarrow b$ is active.

is simultaneous contending traffic on all links in the network. It is important to note that maximizing the link layer throughput through suitable channel assignment may not guarantee maximum network layer throughput (an end-to-end metric), which is a dynamic criterion and depends on the real time traffic conditions in the network. Also, the channel assignments found using this metric may not be optimal for other traffic profiles, e.g., when there is only a few (source, destination) pairs (and corresponding routing paths) in the network.

\section{ILP MODEL - 'A'}

Prior work on fixed channel assignment in cellular networks have used a 'node model', or, in other words, channels are assigned to nodes (base stations). In this paper, we adopt a 'link model', where channels are assigned to links. Consider the simple 6-node grid topology in Figure 2. The solid lines in the figure represent radio links. Existence of a radio link between nodes $i$ and $j$ implies that these nodes are within radio range of each other. For example, the line between nodes 1 and 3 represents that these nodes are within radio range of each other. A pair of nodes which are within radio range of each other are referred to as neighbors. Given a reachability graph $G=(\mathcal{N}, \mathcal{E})$, where $\mathcal{N}$ is the set of nodes and $\mathcal{E}$ is the set of edges (radio links), our objective is to find a channel assignment which would maximize the number of bidirectional links that can be activated simultaneously, subject to interference and other constraints (discussed subsequently).

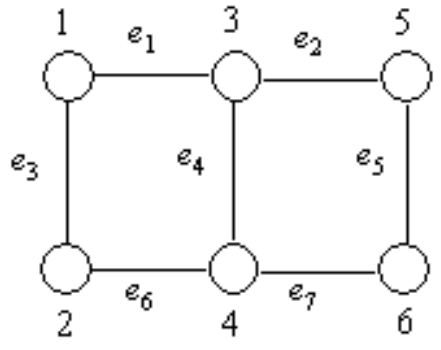

Fig. 2. A 6-node grid topology. The bidirectional edges are numbered $e_{1}$ to $e_{7}$.

Let $\mathcal{F}=[1,2, \cdots F]$ denote the set of available channels, where $F=|\mathcal{F}|$. We assume that the available channels are mutually 'non-overlapping'. Also, let $\mathbf{C}=$ $\left[\mathbf{C}_{e f}: 1 \leq e \leq E, 1 \leq f \leq F\right]$ denote the $E \times F$ binary channel assignment matrix corresponding to the edges in $\mathcal{E}$, such that $\mathbf{C}_{e f}=1$ if edge $e$ is assigned channel $f$. The physical interpretation of $\mathbf{C}_{e f}=1$ is that the end nodes of edge $e$ will use channel $f$ for communicating with each other. For example, if $F=4$ and channel number 2 is assigned to edge $e_{1}$ in Figure 2, the first row of C would be $[0,1,0,0]$, meaning that nodes 1 and 3 (the end nodes of $e_{1}$ ) will use channel 2 to communicate with each other. Finally, we let $\vec{X}=\left[X_{1}, X_{2}, \cdots X_{E}\right]^{T}$ be an $E \times 1$ binary vector such that $X_{e}=1$ if the edge $e$ is part of the optimal solution.

With the above notation in place, we can formally define the objective function for the fixed channel assignment problem with multiple radios (FCA-MR):

$$
\text { FCA-MR: } \quad \text { maximize } \sum_{e \in \mathcal{E}} X_{e}
$$

An implicit assumption in our objective function is that all radio links are potentially traffic-carrying. Or, in other words, if $\mathcal{E}_{t}$ is the set of potentially traffic carrying links, we assume that $\mathcal{E}_{t}=\mathcal{E}$. If, however, $\mathcal{E}_{t} \subset \mathcal{E}$, the summation in (4) can be restricted to the set $e \in \mathcal{E}_{t}$.

In the above form of the objective function, all edges are assumed to be of equal weight. A generalization of the above objective function can be obtained by associating a weight $w_{e}$ with each edge $e$. With an $a$ priori knowledge of the traffic patterns in the network and given a routing algorithm based on a channelunaware metric such as "minimum hop", ${ }^{2}$ the weight $w_{e}$ can be interpreted as the fraction of the total load

\footnotetext{
${ }^{2}$ If the routing metric is channel-aware, such as the WCETT (which is a weighted combination of expected transmission times and maximum channel usage) metric suggested in [15], channel assignment and routing is best treated as a joint optimization problem. However, this is outside the scope of this paper.
} 
offered to the network which is carried on edge $e$. The objective function for the weighted fixed channel assignment problem with multiple radios (WFCA-MR) is therefore:

$$
\text { WFCA-MR: } \operatorname{maximize} \sum_{e \in \mathcal{E}} w_{e} X_{e}
$$

We now discuss the constraints under which the above maximization problem is to be solved.

The first constraint forces all edges in $\mathcal{E}$ to be assigned a channel; i.e.,

$$
\sum_{f} \mathbf{C}_{e f}=1 ; \forall e \in \mathcal{E}
$$

Our second constraint ensures that the number of distinct channels assigned to any node is less than or equal to $K$, the number of radios at each node. As simple as this constraint may sound, modeling it within the framework of ILP model ' $\mathrm{A}$ ' which assigns channels to edges is non-trivial.

Consider, for example, the 6-node network in Figure 2 and the channel assignment matrix shown in (7) for $\mathcal{F}=$ $[1,2,3]$. The edges to which the rows of $\mathbf{C}$ correspond are also shown in (7). The columns of $\mathbf{C}$ correspond to the three available frequency channels.

$$
\mathbf{C}=\left[\begin{array}{lll}
1 & 0 & 0 \\
0 & 1 & 0 \\
0 & 1 & 0 \\
0 & 1 & 0 \\
0 & 0 & 1 \\
0 & 0 & 1 \\
0 & 0 & 1
\end{array}\right] \quad \begin{aligned}
& e_{1}=1 \leftrightarrow 3 \\
& e_{2}=3 \leftrightarrow 5 \\
& e_{3}=1 \leftrightarrow 2 \\
& e_{4}=3 \leftrightarrow 4 \\
& e_{5}=5 \leftrightarrow 6 \\
& e_{6}=2 \leftrightarrow 4 \\
& e_{7}=4 \leftrightarrow 6
\end{aligned}
$$

Take the case of node 3, which has three edges incident on it, $e_{1}, e_{2}$ and $e_{4}$, corresponding to rows 1,2 and 4 of $\mathbf{C}$. The number of radio channels assigned to node 3 is given by: $\left(\mathbf{C}_{11} \vee \mathbf{C}_{21} \vee \mathbf{C}_{41}\right)+\left(\mathbf{C}_{12} \vee \mathbf{C}_{22} \vee \mathbf{C}_{42}\right)+$ $\left(\mathbf{C}_{13} \vee \mathbf{C}_{23} \vee \mathbf{C}_{43}\right)=1+1+0=2$, where $\vee$ denotes the logical OR operation.

Let $\mathbf{Y}=\left[\mathbf{Y}_{n f}: \forall n \in \mathcal{N}, \forall f \in \mathcal{F}\right], 0 \leq \mathbf{Y}_{n f} \leq 1$, be an $N \times F$ matrix such that $\mathbf{Y}_{n f}=1$ if at least one edge incident on node $n$ has been assigned channel $f$. Also, let $\operatorname{rows}(n)$ denote the row indices of $\mathbf{C}$ such that $n$ is an end node in the edges corresponding to rows $(n)$. For example, given the channel assignment matrix (7), $\operatorname{rows}(3)=[1,2,4]$ and $\operatorname{rows}(4)=[4,6,7]$. The number of edges incident on node $n$ is equal to $\mid$ rows $(n) \mid$. Constraint (8) ensures that $\mathbf{Y}_{n f}$ is equal to 1 if at least one edge incident on $n$ is assigned channel $f$. Note that $\mathbf{Y}_{n f}$ is free to take on a value of 1 even if channel $f$ is not assigned to any edge incident on $n$. However, because of the upper bound on the number of channels that can be assigned to node $n$ (9) and our maximization objective, such an assignment would not adversely affect the optimal cost.

$$
\mathbf{Y}_{n f}=\max \left\{\mathbf{C}_{k f}: k \in \operatorname{rows}(n)\right\}, \forall n \in \mathcal{N}, \forall f \in \mathcal{F}
$$

or equivalently,

$$
\mathbf{Y}_{n f} \geq \mathbf{C}_{k f} ; k \in \operatorname{rows}(n), \forall n \in \mathcal{N}, \forall f \in \mathcal{F}
$$

Consider, for example, node 3 in Figure 2 and assume that $f=1$. Using (8), we have the system of inequalities: (i) $\mathbf{Y}_{31} \geq \mathbf{C}_{11}$ (ii) $\mathbf{Y}_{31} \geq \mathbf{C}_{21}$ and (iii) $\mathbf{Y}_{31} \geq \mathbf{C}_{41}$. If channel 1 is assigned to any of the three edges $e_{1}, e_{2}$ and $e_{4}, \mathbf{Y}_{31}$ will be set to 1 .

With the matrix $\mathbf{Y}$ defined as above, the constraint on the maximum number of distinct channels that can be assigned to the edges incident on any node $n$, or equivalently, the number of channels assigned to $n$, can be expressed as:

$$
\sum_{f} \mathbf{Y}_{n f} \leq K ; \forall n \in \mathcal{N}
$$

where $K$ is the number of radios on each node.

Our next constraint set couples the $\left\{X_{e}\right\}$ variables to the $\left[\mathbf{C}_{e f}\right]$ variables and is based on the notion of potentially interfering edges. Given that a particular transmitter and receiver pair are communicating:

- No node within carrier sense range of the transmitter can simultaneously transmit on the same channel, because such a node would defer its transmission upon sensing the channel being busy via the carrier sense (CS) mechanism.

- No node within carrier sense range of the receiver can simultaneously transmit on the same channel because such a transmission would result in a collision at the receiver. Note that the DCF mechanism in 802.11 does not directly prevent these nodes from transmitting and causing a collision, except via the RTS/CTS mechanism. Given the large distances involved in mesh networks, one must expect a relatively high possibility of such hidden nodes, and hence it is likely that RTS/CTS will in fact be employed.

Since transmitters and receivers exchange roles every time an ACK is sent, we are led to the constraint that no edge which is incident on a neighbor of either the transmitter or receiver (which intend to communicate, say, on channel $f$ ) can be simultaneously active on channel $f$. For any bidirected edge $e=(i \leftrightarrow j) \in \mathcal{E}$, the set of its potentially interfering edges, denoted by $\operatorname{IE}(e)$, is therefore:

$$
\begin{aligned}
I E(e)= & \text { all edges incident on }\{n e(i) \backslash j\} \cup \\
& \text { all edges incident on }\{(n e(j) \backslash i)\}
\end{aligned}
$$


where $n e(i)$ is the set of neighbors of node $i$ and ' $U$ ' denotes the union operator. Notice that $\operatorname{IE}(e)$ is essentially the total interference set for the single radio, single channel case. Consider, for example, edge $e_{4}=$ $3 \leftrightarrow 4$ in Figure 2. The set of neighbors of node 3, excluding 4 , is $\{n e(3) \backslash 4\}=\{1,5\}$ and the set of neighbors of node 4 , excluding 3 , is $\{n e(4) \backslash 3\}=\{2,6\}$. From Figure 2, it is seen that the set of all edges incident on node 1 is $\left\{e_{1}, e_{3}\right\}$. Identifying the set of all edges incident on nodes 2,5 and 6 , it can be easily verified that the set of all potentially interfering edges of $e_{4}$ is $\left\{e_{1}, e_{2}, e_{3}, e_{5}, e_{6}, e_{7}\right\}$. Therefore, if edge $e_{4}$ is active, say on channel $f$, none of the other edges in Figure 2 can be simultaneously active on channel $f$.

Given an edge set $\mathcal{E}$, we define an $E \times E$ link interference matrix, LIM, such that the $(a, b)^{t h}(a \neq b)$ element of $\mathbf{L I M}$ is equal to 1 if $\left(e_{a}, e_{b}\right)$ is a potentially interfering pair. All diagonal elements of LIM are equal to 0 . Note that row (column) $a$ of the matrix LIM refers to the edge $e_{a}$.

$$
\mathbf{L I M}_{a b}= \begin{cases}1, & \text { if } e_{b} \in I E\left(e_{a}\right) \\ 0, & \text { otherwise, }\end{cases}
$$

Observe that the matrix LIM is symmetric. For the $3 \times 2$ grid network in Figure 2, the link interference matrix is shown below. The first row and column of the matrix corresponds to edge $e_{1}$, the second row and column corresponds to $e_{2}$, etc.

$$
\left[\begin{array}{lllllll}
0 & 1 & 1 & 1 & 1 & 1 & 1 \\
1 & 0 & 1 & 1 & 1 & 1 & 1 \\
1 & 1 & 0 & 1 & 0 & 1 & 1 \\
1 & 1 & 1 & 0 & 1 & 1 & 1 \\
1 & 1 & 0 & 1 & 0 & 1 & 1 \\
1 & 1 & 1 & 1 & 1 & 0 & 1 \\
1 & 1 & 1 & 1 & 1 & 1 & 0
\end{array}\right]
$$

It is interesting to note that the LIM matrix is essentially the adjacency matrix of the interference graph. Given a reachability graph $G=(\mathcal{N}, \mathcal{E})$ and the LIM matrix, the interference graph, $I(G)$, is a graph whose node set is the edge set of $G$ and two nodes are connected by an edge in $I(G)$ if the corresponding elements in LIM are equal to 1 . Specifically, the nodes $e_{a}$ and $e_{b}\left(e_{a}, e_{b} \in \mathcal{E}\right)$ in $I(G)$ are joined by an edge if $\mathbf{L I M}_{a b}=\mathbf{L I M}_{b a}=1$. See Figure 3 for an illustration.

For networks where all nodes are provided with a single radio, interference issues would prevent any of the edges in $\operatorname{IE}\left(e_{a}\right)$ from coexisting with $e_{a}$. However, for networks with multiple radios, this condition is modified such that "no edge in $\operatorname{IE}\left(e_{a}\right)$ can coexist with $e_{a}$ if they are assigned the same channel". For example, in Figure 2, if $\mathbf{C}_{2,:}=\mathbf{C}_{1, \text { : }}$ (we use the notation $\mathbf{C}_{i, \text { : }}$ to

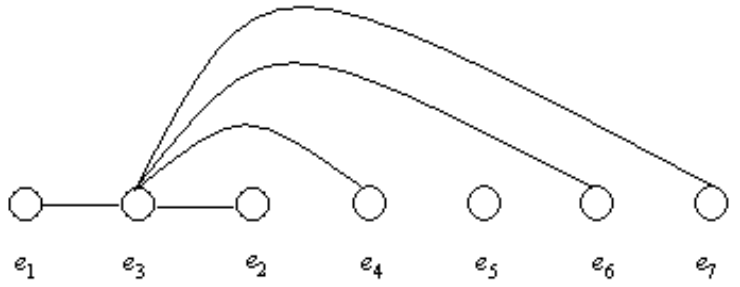

Fig. 3. A partial interference graph corresponding to the link interference matrix (12). The nodes are numbered $e_{1}$ to $e_{7}$, corresponding to the edges in Figure 2. Only the edges which could potentially interfere with $e_{3}$ are shown. For example, nodes $e_{3}$ and $e_{1}$ share an edge since $\mathbf{L I M} \mathbf{I M}_{31}=\mathbf{L} \mathbf{I M}_{13}=1$. Nodes $e_{3}$ and $e_{5}$ do not share an edge since $\mathbf{L I M}_{35}=\mathbf{L I M}_{53}=0$.

denote the $i^{\text {th }}$ row of $\mathbf{C}$ ), both $e_{2}$ and $e_{1}$ cannot be chosen in the optimal solution $\Rightarrow X_{2}+X_{1}$ must be less than or equal to 1 . This restriction does not hold if $\mathbf{C}_{1,:} \neq \mathbf{C}_{2,:}$. Similarly, $e_{2}$ and $e_{4}$ cannot coexist if $\mathbf{C}_{2,:}=\mathbf{C}_{4, \text { : }}$ and the pair $e_{2}$ and $e_{5}$ cannot coexist if $\mathbf{C}_{2,:}=\mathbf{C}_{5,:}$. For the edge $e_{2}$, our channel dependent link assignment constraints are therefore (note that this is an incomplete set; there are other edges which interfere with $e_{2}$ ):

$$
\begin{aligned}
& X_{2}+X_{1} \leq 1, \text { if } \mathbf{C}_{2,:}=\mathbf{C}_{1,:} \\
& X_{2}+X_{4} \leq 1, \text { if } \mathbf{C}_{2,:}=\mathbf{C}_{4,:} \\
& X_{2}+X_{5} \leq 1, \text { if } \mathbf{C}_{2,:}=\mathbf{C}_{5,:}
\end{aligned}
$$

In general, the complete set of channel dependent link assignment constraints is given by:

$$
\begin{aligned}
\forall\left(e_{a}, e_{b}\right) \text { such that } \mathbf{L I M}_{a b} & =1, \\
X_{a}+X_{b} \leq 1, \text { if } \mathbf{C}_{a,:} & =\mathbf{C}_{b,:}
\end{aligned}
$$

It is not hard to see that (14) would lead to duplicate constraints for each interfering pair of edges since the matrix LIM is symmetric. To avoid these duplicate constraints, we rewrite (14) using only the upper triangular portion of LIM above the leading diagonal (the diagonal elements are all 0):

$$
\begin{gathered}
\forall\left(e_{a}, e_{b}\right) \text { such that } U T(\mathbf{L I M})_{a b}=1 \\
X_{a}+X_{b} \leq 1, \text { if } \mathbf{C}_{a,:}=\mathbf{C}_{b,:}
\end{gathered}
$$

where $U T(\mathbf{L I M})$ is the upper triangular portion of LIM above the leading diagonal.

Next, we note that the conditional

$$
X_{a}+X_{b} \leq 1 \text { if } \mathbf{C}_{a,:}=\mathbf{C}_{b,:}
$$

can be expressed as the following system of linear inequalities:

$$
X_{a}+X_{b} \leq 3-\left(\mathbf{C}_{a f}+\mathbf{C}_{b f}\right) ; 1 \leq f \leq F
$$


where $F$ is the number of available frequency channels. Equation (16) represents a system of $F$ equations for each $\left(e_{a}, e_{b}\right)$ pair such that $U T(\mathbf{L I M})_{a b}=1$. It is easy to verify that if edges $e_{a}$ and $e_{b}$ are assigned the same channel, say $f$, one of the inequalities in (16) will reduce to $X_{a}+X_{b} \leq 1$ (the dominating inequality) since $\mathbf{C}_{a f}=$ $\mathbf{C}_{b f}=1$. If they are assigned different channels, say $f_{1}$ and $f_{2}$, the dominating inequality in (16) will be of the form $X_{a}+X_{b} \leq 2$, meaning that both $e_{a}$ and $e_{b}$ are free to be chosen in the optimal solution.

Our next set of constraints are valid inequalities, i.e., they help to improve the LP optimum obtained by relaxing the binary variables, without affecting the set of feasible integer solutions. For any node $n$, let $\left\{X_{e}: n \in e n(e), e \in \mathcal{E}\right\}$ be the set of edges, one of whose end nodes is $n$. The notation $e n(e)$ denotes the end nodes of $e$. Clearly, the number of edges from the set $\left\{X_{e}: n \in e n(e), e \in \mathcal{E}\right\}$ that can be chosen in the optimal solution is limited by the number of distinct channels allocated to node $n$.

$$
\sum_{\substack{e \in \mathcal{E} \\ n \in e n(e)}} X_{e}-\sum_{f} \mathbf{Y}_{n f} \leq 0 ; \forall n \in \mathcal{N}
$$

Consider node 4 in In Figure 2. Assume $F=3$. If the channels assigned to edges $e_{4}, e_{6}$ and $e_{7}$ (which are all incident on node 4) are 2, 3 and 2 respectively, either $e_{4}$ or $e_{7}$ can be chosen in the optimal solution since they are mutually interfering, possibly along with $e_{6}$. That is, $X_{4}+X_{6}+X_{7}$ must be less than or equal to the number of distinct channels assigned to node 4 , which is 2 .

Our final set of constraints are also valid inequalities and is based on the notion of potentially interfering cliques of edges. Given an edge $e$, its interfering clique of edges, denoted by $I C(e)$, is a maximum cardinality subset of $I E(e)$, possibly non-unique, with the property that all edge pairs in the group $\{e \cup I C(e)\}$ are mutually interfering, if assigned the same channel. That is, for any two edges in the group $\{e \cup I C(e)\}$, say $e_{a}$ and $e_{b}, \mathbf{L I M}_{a b}=\mathbf{L I M}_{b a}=1$. From a graph theoretic viewpoint, the line graph corresponding to the edge set $(e \cup I C(e)$ will be fully connected if all edges in $(e \cup I C(e)$ are assigned the same channel.

Consider, for example, edge $e_{1}$ in Figure 2. From (12), $\operatorname{IE}\left(e_{1}\right)=\left\{e_{2}, e_{3}, e_{4}, e_{5}, e_{6}, e_{7}\right\}$. Since $e_{3}$ and $e_{5}$ are non-interfering $\left(\mathbf{L I M}_{35}=\mathbf{L I M}_{53}=0\right), I C\left(e_{1}\right)=$ $\left\{e_{3}, e_{2}, e_{4}, e_{6}, e_{7}\right\}$ or $\left\{e_{5}, e_{2}, e_{4}, e_{6}, e_{7}\right\}$. Irrespective of which subset is chosen, it can be verified that any two edges in the set $\left\{e_{1} \cup I C\left(e_{1}\right)\right\}$ are mutually interfering.

Since all edge pairs in the set $\{e \cup I C(e)\}$ are mutually interfering if assigned the same channel, equation (16) ensures that different channels are assigned to all edges activated from the set, up to a maximum of $F$. For a network with $F$ channels, we therefore have:

$$
X_{e}+\sum_{k \in I C(e)} X_{k} \leq F ; 1 \leq e \leq E
$$

It is interesting to note that the effectiveness of the above inequalities decreases as the size of the maximal clique approaches $F$. For example, if the cardinality of the set $\{e \cup I C(e)\}=F=8$, eqn. (18) merely states that the sum of 8 binary variables is less than or equal to 8 , which is redundant. In Section VI, we provide details of solver computational times for different combinations of $K$ and $F$ which will illustrate that the set of clique inequalities, and indeed, model ' $\mathrm{A}$ ', performs better when the number of available frequency channels is relatively small compared to the maximum clique size of the interference graph. We have also observed that the effectiveness of the above clique inequalities is higher for higher values of $K$. For example, using the LINDO [19] ILP solver, solving model 'A' for a $4 \times 4$ grid, $K=2$ and $F=5$, requires more than 10 hours of solver time, compared to just over a minute for $K=3$ and $F=5$ and only 12 seconds for $K=4$ and $F=5$.

In case $I C(e)$ is non-unique, it is possible to write down the above constraint for all possible combinations of $I C(e)$. For example, we could have used both the inequalities:

$$
\begin{aligned}
& X_{1}+X_{2}+X_{3}+X_{4}+X_{6}+X_{7} \leq F \\
& X_{1}+X_{2}+X_{5}+X_{4}+X_{6}+X_{7} \leq F
\end{aligned}
$$

for the edge $e_{1}$. However, for simplicity, we arbitrarily choose one of the above and include it in the ILP. Equation (18) therefore represents a set of $E$ additional inequalities. Note that (18) may lead to duplicate constraints, which we assume is taken care of by the ILP solver.

Readers familiar with graph theory will recognize that, for any edge $e$, finding the set $\{e \cup I C(e)\}$ is equivalent to finding a constrained maximum clique for the interference graph $I(G)$, with the constraint that the node in $I(G)$ corresponding to $e$ must be chosen. It can be easily shown that the constrained version of the maximum clique problem, like the unconstrained version, is also NP-hard. An exact solution of the constrained maximum interfering clique can be obtained by solving an auxiliary ILP; however for our simulations, we have implemented a variant of the greedy sequential MIN heuristic [20] for the maximal independent set/maximal clique problem ${ }^{3}$, as outlined in Figure 4.

\footnotetext{
${ }^{3}$ Solving the maximal clique problem on the graph $G$ is equivalent to solving the maximum independent set problem on the graph $\bar{G}$ where $\bar{G}$ is the complement of $G$.
} 
Input: $I(G)=(\mathcal{N}(I(G)), \mathcal{E}(I(G)))$, an edge $e \in \mathcal{E}$, $I E(e)$ and LIM

Output: The set $C=\{e \cup I C(e)\}$

/* Find the degree vector of the nodes in $I(G)$ which correspond to the edges in $\operatorname{IE}(e) *$ /

$\operatorname{deg}=\sum_{b} \mathbf{L I M}_{a b} ; e_{a} \in \operatorname{IE}(e)$

/* Initialize $C$ with $e$ */

$C \leftarrow\{e\} ;$

flag $=1$

while (flag)

- Find the edge $e_{m} \in I E(G)$ which has the highest node degree in $I(G)$.

- If there are multiple edges which have the highest node degree in $I(G)$, choose one randomly.

- /* Delete $e_{m}$ from $I E(e) *$ / $I E(e) \leftarrow I E(e) \backslash e_{m} ;$

- Find all other nodes $\left\{e_{n}\right\} \in I E(e)$ which are not connected to $e_{n}$; i.e., $\mathbf{L I M}_{m n}=0$.

- I* Delete $\left\{e_{n}\right\}$ from $I E(e) * /$ $I E(e) \leftarrow I E(e) \backslash\left\{e_{n}\right\}$;

- /* Recompute the degree vector */ $d e g=\sum_{b} \mathbf{L I M}_{a b} ; e_{a} \in I E(e)$

- /* Update $C *$ / $C \leftarrow\left\{C \cup e_{m}\right\}$

- /* Terminate if $I E(e)$ is empty */

if $(\operatorname{IE}(e)==\emptyset)$ flag $=0$;

end

end

Fig. 4. A pseudo-code of the greedy sequential heuristic for computing a constrained maximal interfering clique of an edge $e \in \mathcal{E}$, or equivalently, the node $e \in \mathcal{N}(I(G)$ ). Recall that the edges in $\mathcal{E}$ are nodes in the interference graph $I(G)$.

Figure 5 summarizes ILP model 'A' for the WFCAMR problem. The number of binary variables in this model is $E(F+1)$ and the number of continuous variables is $N F$. The number of constraints is equal to $2(E+N)+F \sum_{n} \operatorname{deg}_{n}+\sum_{a, b} U T(\mathbf{L I M})_{a b}$, where $\operatorname{deg}_{n}$ is the degree of node $n$.

\section{ILP MODEL - 'B'}

In this section, we discuss another ILP model which is based on a transformation of the reachability graph, $G=(\mathcal{N}, \mathcal{E})$. Let us denote the transformed graph by $G^{\prime}=\left(\mathcal{N}, \mathcal{E}^{\prime}\right)$, where $E^{\prime}=\left|\mathcal{E}^{\prime}\right|=E \times F$. Recall that $F$ is the number of available frequency channels and $N$ and $E$ are the number of nodes and bidirected edges in $G$. $\operatorname{maximize} \sum_{e} w_{e} X_{e}$

subject to

$$
\begin{aligned}
0 \leq \mathbf{Y}_{n f} \leq 1 ; \forall n \in \mathcal{N}, \forall f \in \mathcal{F} \\
\mathbf{C}_{e f} \in\{0,1\} ; \forall e \in \mathcal{E}, \forall f \in \mathcal{F} \\
X_{e} \in\{0,1\} ; \forall e \in \mathcal{E} \\
\sum_{f} \mathbf{C}_{e f}=1 ; \forall e \in \mathcal{E} \\
\mathbf{C}_{k f}-\mathbf{Y}_{n f} \leq 0 ; \quad k \in \operatorname{rows}(n), \\
\sum_{f} \mathbf{Y}_{n f} \leq K ; \forall n \in \mathcal{N}, \forall f \in \mathcal{F} \\
X_{a}+X_{b}+\left(\mathbf{C}_{a f}+\mathbf{C}_{b f} \leq 3 ; \quad \forall f \in \mathcal{F}, \forall\left(e_{a}, e_{b}\right)\right. \text { such } \\
\text { that } U T(\mathbf{L I M})_{a b}=1 \\
\sum_{\substack{e \in \mathcal{E} \\
n \in e n(e)}} \sum_{f=1}^{F} \mathbf{Y}_{n f} \leq 0 ; \forall n \in \mathcal{N} \\
X_{e}+\sum_{k \in I C(e)} X_{k} \leq F ; \forall e \in \mathcal{E}
\end{aligned}
$$

Fig. 5. ILP model 'A' for the weighted fixed channel assignment problem in wireless networks with multiple radios. The notation $U T(\mathbf{L I M})$ represents the upper triangular portion of the matrix LIM, excluding the leading diagonal. The notation $\operatorname{rows}(n)$ denotes the row indices of $\mathbf{C}$ such that $n$ is an end node in the edges corresponding to rows $(n)$.

Figure 6 shows a transformation of the reachability graph shown in Figure 2 for $F=3$. Note that each edge in Figure 2 has been triplicated in Figure 6 since $F=3$. In general, each bidirected edge $e \in \mathcal{E}$ leads to $F$ copies in $\mathcal{E}^{\prime}$, numbered $(e, 1),(e, 2), \cdots(e, F)$. Let us denote this set of edges by $\left\{e^{\prime}\right\}$; i.e., $e \in \mathcal{E} \mapsto\left\{e^{\prime}\right\} \in \mathcal{E}^{\prime}$ where $\left\{e^{\prime}\right\}=[(e, 1),(e, 2), \cdots(e, F)]$. The set of all edges in $G^{\prime}$ is therefore: $\mathcal{E}^{\prime}=\{(e, f): 1 \leq e \leq E, 1 \leq f \leq F\}$.

$$
\left[\begin{array}{c}
e_{1} \\
e_{2} \\
\vdots \\
e_{E}
\end{array}\right] \in G \mapsto\left[\begin{array}{cccc}
e_{11} & e_{12} & \cdots & e_{1 F} \\
e_{11} & e_{12} & \cdots & e_{1 F} \\
\vdots & \vdots & \ddots & \vdots \\
e_{E 1} & e_{E 2} & \cdots & e_{E F}
\end{array}\right] \in G^{\prime}
$$

Let $\mathbf{C}=\left[\mathbf{C}_{e f}: 1 \leq n \leq N, 1 \leq f \leq F\right]$ be the binary $E \times F$ channel assignment matrix such that $\mathbf{C}_{e f}=1$ if edge $e \in \mathcal{E}$ is assigned channel $f$.

Also, let $\mathbf{X}=\left[\mathbf{X}_{e f}: 1 \leq e \leq E, 1 \leq p \leq F\right]$ denote the $E \times F$ binary matrix such that $\mathbf{X}_{e f}=1$ if the edge $(e, f)$ is chosen in the optimal solution. Our objective 


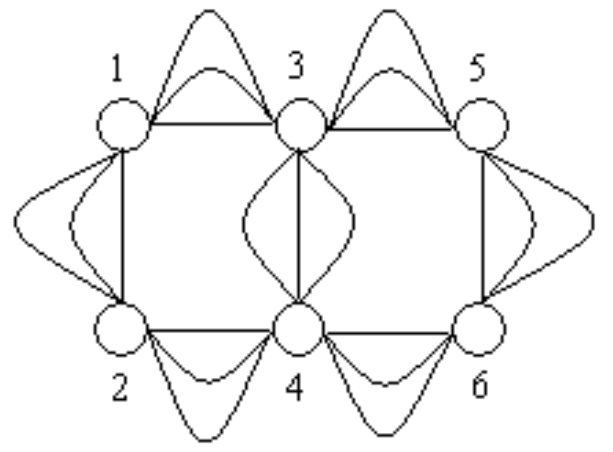

Fig. 6. A transformation of the reachability graph shown in Figure 2, for $F=3$. Each edge in Figure 2 has been triplicated. Specifically, edge $e$ in Figure 2 maps to the set of three edges $\{(e, 1),(e, 2),(e, 3)\}$.

function in ILP model ' $\mathrm{B}$ ' is:

$$
\text { maximize } \sum_{e=1}^{E} \sum_{f=1}^{F} w_{e} \mathbf{X}_{e f}
$$

Note that the weights $\left\{w_{e}\right\}$ are independent of $f$; i.e., given an edge $e \in \mathcal{E}$ and a corresponding weight $w_{e}$, all edges in the group $\left\{e^{\prime}\right\}=[(e, 1),(e, 2), \cdots(e, F)] \in \mathcal{E}^{\prime}$ share the same weight $w_{e}$. The constraints under which the above maximization problem is to be solved are discussed below.

As in model ' $\mathrm{A}$ ', the first constraint forces all edges in $\mathcal{E}$ to be assigned a channel; i.e.,

$$
\sum_{f} \mathbf{C}_{e f}=1 ; \forall e \in \mathcal{E}
$$

The second constraint ensures that an edge $(e, f)$ is chosen in the optimal solution only if edge $e \in \mathcal{E}$ is assigned channel $f$, i.e., if $\mathbf{C}_{e f}=1$ :

$$
\mathbf{X}_{e f}-\mathbf{C}_{e f} \leq 0 ; \forall e \in \mathcal{E}, \forall f \in \mathcal{F}
$$

Note that constraints (20) and (21) together implies:

$$
\sum_{f} \mathbf{X}_{e f} \leq \sum_{f} \mathbf{C}_{e f}=1 ; \forall e \in \mathcal{E}
$$

Our third constraint ensures that the number of channels assigned to any node is less than or equal to $K$, the number of radios at each node. As in model 'A', we define an auxiliary $N \times F$ matrix $\mathbf{Y}=\left[\mathbf{Y}_{n f}: \forall n \in\right.$ $\mathcal{N}, \forall f \in \mathcal{F}], 0 \leq \mathbf{Y}_{n f} \leq 1$, such that $\mathbf{Y}_{n f}=1$ if at least one edge incident on node $n$ have been assigned channel $f$.

$\mathbf{Y}_{n f}=\max \left\{\mathbf{C}_{k f}: k \in \operatorname{rows}(n)\right\}, \forall n \in \mathcal{N}, \forall f \in \mathcal{F}$

or equivalently,

$\mathbf{Y}_{n f} \geq \mathbf{C}_{k f} ; k \in \operatorname{rows}(n), \forall n \in \mathcal{N}, \forall f \in \mathcal{F}$
The constraint on the maximum number of channels that can be assigned to the edges incident on any node $n$ can be expressed as:

$$
\sum_{f} \mathbf{Y}_{n f} \leq K ; \forall n \in \mathcal{N}
$$

Our fourth set of constraints define pairwise edge exclusions based on the LIM matrix. Because of the mapping $e \in \mathcal{E} \mapsto\{(e, 1),(e, 2), \cdots(e, F)\} \in \mathcal{E}^{\prime}$, the interference constraints can be simply expressed as:

$$
\begin{aligned}
& \forall\left(e_{a}, e_{b}\right) \text { such that } U T(\mathbf{L I M})_{a b}=1 \\
& \quad \mathbf{X}_{a f}+\mathbf{X}_{b f} \leq 1 ; \forall f \in \mathcal{F}
\end{aligned}
$$

Equation (24) specifies that a pair of potentially interfering edges cannot both be part of the optimal solution if they are assigned the same channel.

Besides the pairwise edge exclusion constraints, we can also use an aggregated version for each edge $e \in \mathcal{E}$. Let $\left\{k: \forall k\right.$ such that $\left.\mathbf{L I M}_{e k}=1\right\}$ denote the set of potentially interferer edges of $e$. Consider row 1 of the LIM matrix (12), corresponding to edge $e_{1}$. The set of interferer edges of $e_{1}$ is $\{k\}=\left\{e_{2}, e_{3}, e_{4}, e_{5}, e_{6}, e_{7}\right\}$. For any $f$, if $X_{1 f}=1$, none of its interferer edges can be chosen if they are assigned the same channel. That is,

$$
X_{2 f}+X_{3 f}+X_{4 f}+X_{5 f}+X_{6 f}+X_{7 f}
$$

must be equal to 0 if $X_{1 f}=1$. For any edge $e$, this can be represented as:

$$
\sum_{\substack{k \in \mathcal{E} \\ \mathbf{L I M}_{e k}=1}} \mathbf{X}_{k f}-|k| \cdot\left(1-\mathbf{X}_{e f}\right) \leq 0 ; \forall f \in \mathcal{F}
$$

If $\mathbf{X}_{e f}=1$, the leftmost summation term is forced to 0 . Otherwise, the above constraint is redundant since the $\mathbf{X}_{e f}$ variables are binary. Note that (25) represents a set of $F$ inequalities for each edge $e \in \mathcal{E}$.

The first set of valid inequalities discussed in the previous section can also be extended straightforwardly.

$$
\sum_{\substack{e \in \mathcal{E} \\ n \in e n(e)}} \sum_{f} \mathbf{X}_{e f}-\sum_{f} \mathbf{Y}_{n f} \leq 0 ; \forall n \in \mathcal{N}
$$

For any node $n$, constraint (26) forces the number of edges incident on $n$ that can be chosen in the optimal solution to be less than the number of distinct channels assigned to $n$.

Finally, we discuss the clique inequalities. For some $e$ and a specific channel $f$, at most one edge in the set $\{(e, f) \cup(k, f): k \in I C(e)\}$ can be chosen, or more formally:

$$
\mathbf{X}_{e f}+\sum_{k \in I C(e)} \mathbf{X}_{k f} \leq 1 ; \forall f \in \mathcal{F}, \forall e \in \mathcal{E}
$$


It is important to note that the r.h.s of the above inequality is 1 , compared to $F$ in model 'A'. Because the r.h.s of (27) is independent of $F$, the effectiveness of these clique inequalities does not decrease (in fact, it increases) as the size of the maximum clique increases, as was the case in model ' $\mathrm{A}$ '. The impact of this on solver times for ILP models 'A' and 'B' will be apparent from Section VI.

Figure 7 summarizes ILP model ' $\mathrm{B}$ ' for the WFCAMR problem. The number of binary variables in this model is $2 E F$ (compared to $E(F+1)$ for model 'A') and the number of continuous variables is $N F$. The number of constraints is equal to $2 N+E(1+2 F)+F \sum_{n} d e g_{n}+$ $\sum_{a, b} U T(\mathbf{L I M})_{a b}$, where $\operatorname{deg}_{n}$ is the degree of node $n$.

While models ' $\mathrm{A}$ ' and 'B' may appear to be similar, except for the clique inequalities, simulations on various grid topologies suggest that the polyhedron defined by ILP ' $\mathrm{B}$ ' is probably tighter than the polyhedron defined by ILP 'A'. In our simulations on $4 \times 4$ topologies, the solutions obtained from model ' $\mathrm{B}$ ' turned out to be integer even when the $\mathbf{C}$ variables were relaxed (i.e., when they are allowed to take continuous values between 0 and 1.). This is not the case for model 'A. Relaxing the $\mathbf{C}$ variables in model ' $\mathrm{A}$ ' led to fractional values for both $\mathbf{C}$ and $\mathbf{Y}$ variables. Whether the naturally occurring integer solutions with $\mathbf{C}$ relaxed is specific to the grid topologies considered or is generally true requires an analysis of the polyhedral aspects of models ' $A$ ' and ' $B$ '. We plan on undertaking such a study in the future.

\section{Simulation Results}

In this section, we present the ILP solutions for regular grid topologies of size $4 \times 4,5 \times 5$ and $6 \times 6$. Before presenting the results however, we undertake a brief qualitative discussion of the channel assignment problem on grid topologies. Consider the 8 edge subset of a grid shown in Figure 8. It is easy to verify that these edges all potentially interfere with each other, i.e., the corresponding nodes in the interference graph form a clique (this clique is in fact maximal). Therefore on any subset of 8 edges of this form, no more than $F$ edges can be active simultaneously, where $F$ is the total number of available channels. For the rest of this discussion, we will restrict ourselves to the case where $F=3$ and the number of radios $K=2$ (which would be a typical scenario in $802.11 \mathrm{~b} / \mathrm{g}$ meshes). Now note that the square lattice can be tiled with sets of edges of the form of the maximal clique, as seen in Figure 9. The latter figure also shows an assignment of 3 channels to edges in the clique which ensures that no two links with the same channel assignment interfere with each other. Therefore with 3 available channels and 2 radios per node, the $\operatorname{maximize} \sum_{e=1}^{E} \sum_{f=1}^{F} w_{e} \mathbf{X}_{e f}$

subject to

$$
\begin{aligned}
& 0 \leq \mathbf{Y}_{n f} \leq 1 ; \forall n \in \mathcal{N}, \forall f \in \mathcal{F} \\
& \mathbf{C}_{e f} \in\{0,1\} ; \forall e \in \mathcal{E}, \forall f \in \mathcal{F} \\
& \mathbf{X}_{e f} \in\{0,1\} ; \forall e \in \mathcal{E}, \forall f \in \mathcal{F} \\
& \sum_{f} \mathbf{C}_{e f}=1 ; \forall e \in \mathcal{E} \\
& \mathbf{C}_{k f}-\mathbf{Y}_{n f} \leq 0 ; k \in \operatorname{rows}(n), \\
& \forall n \in \mathcal{N}, \forall f \in \mathcal{F} \\
& \sum_{f} \mathbf{Y}_{n f} \leq K ; \forall n \in \mathcal{N} \\
& \mathbf{X}_{a f}+\mathbf{X}_{b f} \leq 1 ; \forall f \in \mathcal{F}, \forall\left(e_{a}, e_{b}\right) \\
& \text { s.t. } U T(\mathbf{L I M})_{a b}=1 \\
& \sum_{k \in \mathcal{E}} \mathbf{X}_{k f}-|k| \cdot\left(1-\mathbf{X}_{e f}\right) \leq 0 ; \forall f \in \mathcal{F}, \forall e \in \mathcal{E} \\
& \mathbf{L I M}_{e k}=1 \\
& \sum_{\substack{e \in \mathcal{E} \\
n \in e n(e)}} \sum_{f} \mathbf{X}_{e f}-\sum_{f} \mathbf{Y}_{n f} \leq 0 ; \forall n \in \mathcal{N} \\
& \mathbf{X}_{e f}+\sum_{k \in I C(e)} \mathbf{X}_{k f} \leq 1 ; \forall f \in \mathcal{F}, \forall e \in \mathcal{E}
\end{aligned}
$$

Fig. 7. ILP model ' $B$ ' for the weighted fixed channel assignment problem in wireless networks with multiple radios. The notation $U T(\mathbf{L I M})$ represents the upper triangular portion of the matrix LIM, excluding the leading diagonal. The notation rows $(n)$ denotes the row indices of $\mathbf{C}$ such that $n$ is an end node in the edges corresponding to $\operatorname{rows}(n)$.

fraction of all edges that can be active simultaneously approaches $3 / 8(37.5 \%)$ asymptotically as the number of nodes grows large.

However, for the finite grids that we will consider in this section, a higher fraction of edges can be simultaneously active; this is achieved by a suitable assignment to the border edges in the graph. For the $4 \times 4$ case, the number of edges in the optimal solution, as found by the ILP, was 12 (or $50 \%$ of all edges). In the $5 \times 5$ and $6 \times 6$ cases, 18 and 27 edges respectively were in the optimal solution, corresponding in both cases to $45 \%$ of all edges. Figure 10 illustrates the channel assignment for the $6 \times 6$ grid. In this figure, each edge is labeled with the channel assigned to it, and edges that are part of the optimal solution are drawn in bold.

\section{A. Detailed results for the $4 \times 4$ grid}

In this section, we provide detailed simulation results for the $4 \times 4$ grid. Table I lists the solver times for 


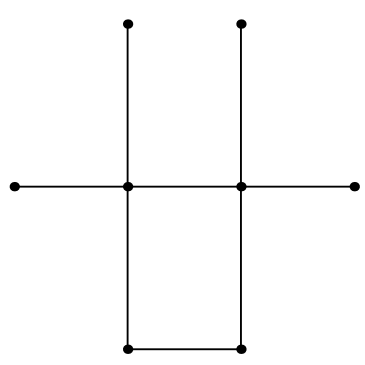

Fig. 8. Maximum clique of 8 edges that all interfere with each other.

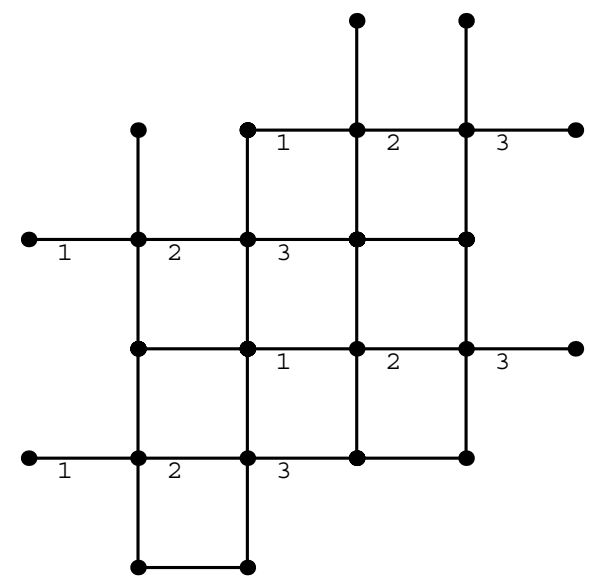

Fig. 9. Illustrating tiling of lattice with cliques.

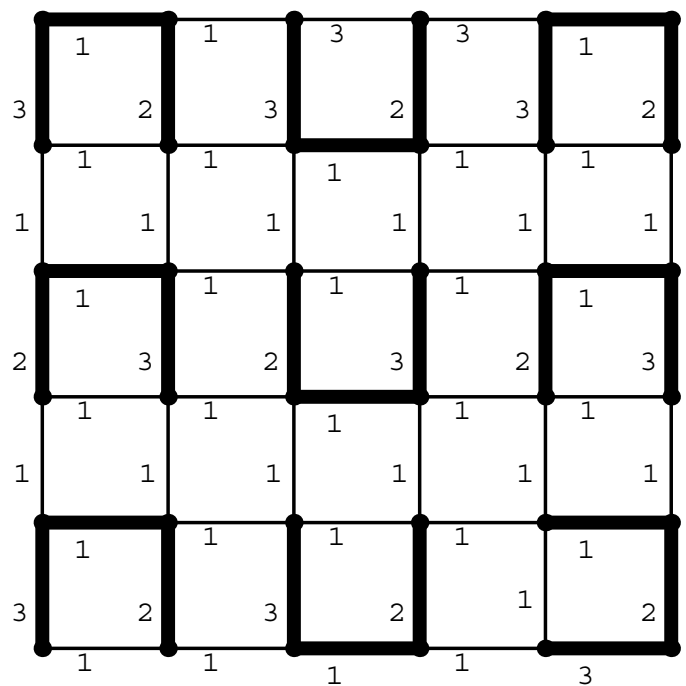

Fig. 10. With 3 channels and 2 radios, 27 edges can be active simultaneously in the $6 \times 6$ grid. Edges which are active are shown in bold.

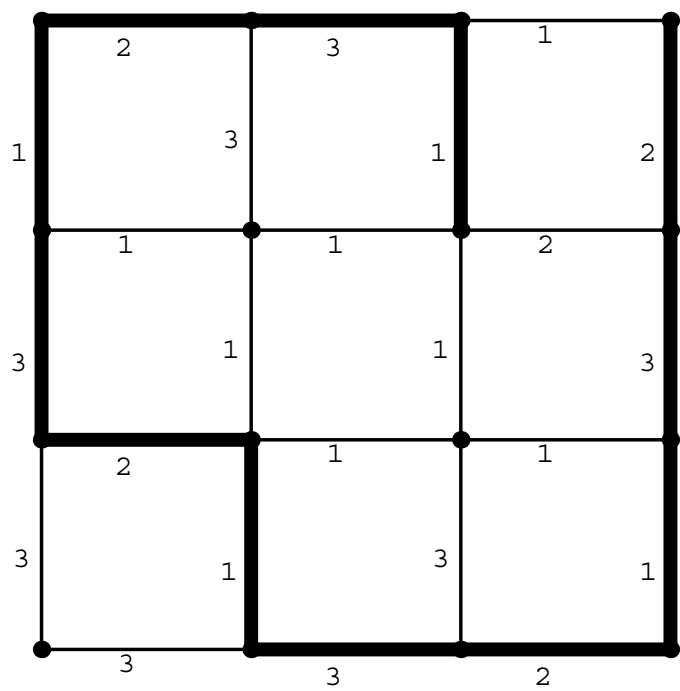

Fig. 11. With 3 channels and 2 radios, 12 edges can be active simultaneously in the $4 \times 4$ grid. Edges which are active are shown in bold.

model 'A' and optimal ILP costs with different $K$ and $F$ combinations. All simulations were conducted using the LINDO mixed integer linear programming solver [19].

We see from the results that the number of active links in the optimal solution grows from 4, if there is only one channel available, to 24 (i.e. all links in the network) when there are 4 radios and 8 channels available. Figure 11 shows the channel assignment for the same 2 radio and 3 channel case that we considered earlier for the larger grids.

It can be seen from Table I that, typically, for a fixed $K$, the solution time increases with $F$, though there are some exceptions. For example, the solver time for $K=4$ and $F=7$ is almost 30 times that for $K=4$ and $F=8$. The most striking observation, however, is the huge jump in solver time from $F=3$ to $F=4$ for $K=2$ (almost 450 times) and, to a certain extent, from $F=4$ to $F=5$ (almost 15 times). We have tested the computational efficiency of model ' $\mathrm{B}$ ' for some of the cases where model ' $\mathrm{A}$ ' required long solver times. A comparison is provided in Table II. Observe that model ' $\mathrm{B}$ ' requires less than $50 \%$ solver time than model ' $\mathrm{A}$ ' for all these cases.

\section{CONCLUSION}

We have presented two ILP formulations for the fixed channel assignment problem in multi-radio meshes with the goal of maximizing the number of simultaneous transmissions in the network. Our numerical results illustrate the benefits obtained by increasing the number of radios per node and the number of available channels in the network. However, realizing the full potential of 
TABLE I

Solver times and optimal ILP costs (maximum number of links that can be active simultaneously) for simulations conducted on a $4 \times 4$ grid with different $K$ and $F$ combinations. The solution times listed are for model 'A'.

\begin{tabular}{|c|c|c|c|}
\hline$K$ & $F$ & Time (secs) & Opt. Cost \\
\hline $1 / 2 / 3 / 4$ & 1 & $<1$ & 4 \\
\hline \multirow{3}{*}{2} & 2 & 1 & 8 \\
\cline { 2 - 4 } & 3 & 6 & 12 \\
\cline { 2 - 4 } & 4 & 2727 & 14 \\
\cline { 2 - 4 } & 5 & 39363 & 14 \\
\hline \multirow{4}{*}{3} & 2 & 1 & 8 \\
\cline { 2 - 4 } & 3 & 4 & 12 \\
\cline { 2 - 4 } & 4 & 11 & 16 \\
\cline { 2 - 4 } & 5 & 72 & 20 \\
\cline { 2 - 4 } & 6 & 37 & 21 \\
\hline \multirow{4}{*}{4} & 2 & 2 & 8 \\
\cline { 2 - 4 } & 3 & 2 & 12 \\
\cline { 2 - 4 } & 4 & 13 & 16 \\
\cline { 2 - 4 } & 5 & 12 & 20 \\
\cline { 2 - 4 } & 6 & 14 & 21 \\
\cline { 2 - 4 } & 7 & 947 & 22 \\
\cline { 2 - 4 } & 8 & 35 & 24 \\
\hline
\end{tabular}

TABLE II

Comparison of solver times for models 'A' and 'B', $4 \times 4$ grid.

\begin{tabular}{|c|c|c|c|}
\hline$K$ & $F$ & Time (secs.) ILP 'A' & Time (secs.) ILP 'B' \\
\hline 2 & 4 & 2727 & 1171 \\
\hline 2 & 5 & 39363 & 15631 \\
\hline 3 & 5 & 72 & 28 \\
\hline 4 & 7 & 947 & 57 \\
\hline
\end{tabular}

multiple radios and multiple channels will require the use of an appropriate routing algorithm which can use these channel assignments along with information about the traffic pattern optimally. Ongoing work focuses on studying the choice of routing algorithm and evaluating the impact of the channel assignment and routing algorithm on the throughput and delay characteristics of the network. In particular, we are interested in studying the use of appropriate weights on the links to tune the channel assignment based on knowledge of the expected traffic patterns. We are also investigating heuristic algorithms for the channel assignment problem; the results in this paper will be used as benchmarks against which the performance of any heuristic can be compared.

\section{REFERENCES}

[1] Barry Leiner, Donald Nielson, and Fouad Tobagi, "Issues in packet radio network design," Proceedings of the IEEE, vol. 75, no. 1, pp. 6-20, January 1987.

[2] Fouad Tobagi, "Modeling and performance analysis of multihop packet radio networks," Proceedings of the IEEE, vol. 75, no. 1 , pp. 135-155, January 1987.
[3] Israel Cidon and Moshe Sidi, "Distributed assignment algorithms for multihop packet radio networks," IEEE Transactions on Computers, vol. 38, no. 10, pp. 1353-1361, October 1989.

[4] B. Hajek and G. Sasaki, "Link scheduling in polynomial time," IEEE Transactions on Information Theory, vol. 34, no. 5, pp. 910-917, September 1988.

[5] R. G. Ogier, "A decomposition method for optimal scheduling," Proceeding of 24th Annual Allerton Conference, pp. 822-823, October 1986.

[6] R. Ramaswami and K. K. Parhi, "Distributed scheduling of broadcasts in a radio network," Proc. 8th Annual Joint Conf. of IEEE Computer and Communication Societies INFOCOM, 1989, pp. 497-504, 1989.

[7] A. Ephremides and T. V. Truong, "Scheduling broadcasts in multihop radio networks," IEEE Transactions on Communications, vol. 38, pp. 456-461, April 1990.

[8] Arunabha Sen and Mark L. Huson, "A new model for scheduling packet radio networks," Wireless Networks, vol. 3, pp. 7181, 1997.

[9] N. Jain, S. Das, and A. Nasipuri, "A multichannel CSMA MAC protocol with receiver-based channel selection for multihop wireless networks," IEEE Wireless Communications and Networking Conference (WCNC), September 1999.

[10] N. Jain, S. Das, and A. Nasipuri, "Multichannel csma with signal power-based channel selection for multihop wireless networks," Proc. of IEEE Vehicular Technology Conference (VTC), September 2000.

[11] Jungmin So and Nitin H. Vaidya, "Multichannel mac for ad hoc networks: Handling multichannel hidden terminals using a single transceiver," ACM International Symposium on Mobile Ad Hoc Networking and Computing (MobiHoc), May 2004.

[12] Jungmin So and Nitin H. Vaidya, "A routing protocol for utilizing multiple channels in multi-hop wireless networks with a single transceiver," Tech. Report University of Illinois at Urbana-Champaign, Ocober 2004.

[13] Ranveer Chandra Paramvir Bahl and John Dunagan, "Sch: Slotted seeded channel hopping for capacity improvement in ieee 802.11 ad-hoc wireless networks," in ACM Mobicom, 2004.

[14] A. Adya, P. Bahl, J. Padhye, A. Wolman, and L. Zhou, "A multiradio unification protocol for IEEE 802.11 wireless networks," Microsoft Technical Report, vol. MSR-TR-2003-44, July 2003.

[15] Richard Draves, Jitendra Padhye, and Brian Zill, "Routing in multi-radio, multi-hop wireless mesh networks," in MobiCom '04: Proceedings of the 10th annual international conference on Mobile computing and networking, New York, NY, USA, 2004, pp. 114-128, ACM Press.

[16] P. Kyasanur and N. Vaidya, "Routing and interface assignment in multi-channel multi-interface wireless networks," University of Illinois at Urbana-Champaign Technical Report, Oct. 2004.

[17] Ashish Raniwala and Tzi cker Chiueh, "Architecture and algorithms for an IEEE 802.11-based multi-channel wireless mesh network," INFOCOM 2005.In Proceedings of Twentyfourth Annual Joint Conference of the IEEE Computer and Communications Societies. IEEE, 2005.

[18] Ashish Raniwala, Kartik Gopalan, and Tzi cker Chiueh, "Centralized channel assignment and routing algorithms for multichannel wireless mesh networks," SIGMOBILE Mob. Comput. Commun. Rev., vol. 8, no. 2, pp. 50-65, 2004.

[19] "LINDO MILP solver," http://www.lindo.com.

[20] O. Murphy, "Lower bounds on the stability number of graphs computed in terms of degrees," Discrete Mathematics, vol. 90, pp. 207-211, 1991. 